\title{
THE DYNAMIC ROLLING RADIUS OF A PNEUMATIC TYRE ON HARD TERRAINS ${ }^{\#}$
}

\author{
Herman A. Hamersma ${ }^{1 *}$, Theunis R. Botha ${ }^{2}$ \& P. Schalk Els ${ }^{3}$ \\ Department of Mechanical and Aeronautical Engineering, University of Pretoria, \\ Hatfield 0028, South Africa \\ 1'hermanh@up.ac.za, ${ }^{2}$ theunis.botha@up.ac.za, ${ }^{3}$ schalk.els@up.ac.za
}

*Corresponding author

${ }^{1}$ Author was enrolled for a PhD (Mechanical Engineering) in the Department of Mechanical and Aeronautical Engineering, University of Pretoria, South Africa

${ }^{2}$ Dr Theunis Botha is a Senior Lecturer in the Department of Mechanical and Aeronautical Engineering, University of Pretoria, South Africa

${ }^{3}$ Prof Schalk Els is a Full Professor in the Department of Mechanical and Aeronautical Engineering, University of Pretoria, South Africa

\begin{abstract}
Extensive research was done on the rolling radius of a tyre from the 1960s to the 1980s, specifically looking at the tractive performance of tractors and agricultural vehicles. The research was limited to investigating slip conditions at low vehicle speed where it was safe to assume that the rolling radius is static or quasi-static. This paper revisits some of these investigations, and looks at the validity of the static or quasi-static rolling radius assumption on hard, high friction and uneven surfaces at higher speeds. The aim is to establish whether the rolling radius defined under static conditions can be used to estimate tyre contact patch velocity, a state needed to determine longitudinal tyre slip. Longitudinal tyre slip plays a crucial role in the performance of advanced driver assist systems. It is known that these systems' performance decreases on rough roads when the static rolling radius assumption may become inaccurate.
\end{abstract}

Keywords: Kinematic rolling radius; kinetic rolling radius; contact patch velocity; digital image correlation; wheel force transducer measurements; tyre modelling

\section{NOMENCLATURE}

\begin{tabular}{|l|l|}
\hline Symbol & Description \\
\hline Roman symbols \\
\hline$F_{x}$ & Longitudinal tyre force \\
\hline
\end{tabular}

\footnotetext{
\# This paper is a revised and expanded version of a paper entitled 'Kinetic vs. kinematic roll radius on rough roads' presented at 18th International Conference of the ISTVS, Seoul, Korea, 22-24 September 2014.
} 


\begin{tabular}{|c|l|}
\hline$F_{Z}$ & Vertical tyre force \\
\hline$I_{o}$ & Rigid disc moment of inertia \\
\hline$M_{O}$ & Moment about point $\mathrm{O}$ \\
\hline$M_{y}$ & Brake torque \\
\hline$r$ & Rolling radius \\
\hline$r_{\text {kinematic }}$ & Kinematic rolling radius \\
\hline$r_{\text {kinetic }}$ & Kinetic rolling radius \\
\hline$\Delta t$ & Change in time \\
\hline$v$ & Longitudinal vehicle speed \\
\hline$v_{c p}$ & Contact patch speed \\
\hline Greek symbols & \\
\hline$\dot{\theta}$ & Wheel angular velocity \\
\hline$\ddot{\theta}$ & Wheel angular acceleration \\
\hline$\lambda$ & Longitudinal slip \\
\hline$\rho$ & Tyre vertical force offset from wheel centreline \\
\hline
\end{tabular}

\section{INTRODUCTION}

With the improvements in vehicle technology experienced in the last few decades, vehicles with high centres of gravity (typically associated with the agricultural, mining and military sectors) have been able to achieve ever increasing speeds. Hand-in-hand with an increase in speed is an increased demand in the safety features that are required in these vehicles. As a result, advanced driver assist systems (ADAS) such as traction control (TC), electronic stability control (ESC) and anti-lock braking systems (ABS) have become prevalent throughout the industry (Breuer and Bill, 2008). Although these ADAS systems have been implemented successfully on passenger vehicles traversing paved roads and highways, the implementation of these systems on off-road vehicles driving on uneven and off-road terrains have left much to be desired. Hamersma and Els (2014) found that there is a significant reduction in the ABS braking performance of a sports utility vehicle (SUV) on a Belgian paving, an undulating and rough road, when compared to the braking performance on a smooth road.

Many of these ADAS algorithms rely on some form of longitudinal tyre slip estimation, a typical example is the Bosch ABS algorithm (Siegert et al., 1999). With the advent of SportsUtility-Vehicles (SUVs), these driver assist systems have been applied to vehicles that have the ability to traverse rough terrain and severely undulating roads at relatively high speeds. Longitudinal slip estimation almost always relies on estimating (or assuming) an effective rolling radius and hence the investigation of the rolling radius on various road surfaces has been a topic of discussion and scientific investigation for many years. A significant amount of work was done specifically in the 1980s by Upadhyaya et al. (1988), specifically looking at the tractive performance of agricultural vehicles. These studies investigated the effect of the test procedure and the interpretation of rolling radius and motion resistance. The rolling radius used in these studies is defined under an explicitly stated zero-condition. The "true rolling radius" and "true slip" were then determined empirically and a nonlinear regression was used to fit a curve through the obtained test data. The shortcoming with these approaches to the rolling radius is the assumption that the rolling radius is a constant value that can be determined under quasi-static conditions (as done by Kiss (2003)) or a large amount of empirical data is needed 
to determine the "true rolling radius" or "true slip" as done by Upadhyaya et al. (1988). The nature of these studies was also limited to soft, deformable terrains where the dynamics of the manoeuvre is of less importance and the quasi-static approach used by Kiss (2003) may yield acceptable results.

Kiss (2003) investigated three radii of agricultural tyres, namely the distance between the bottom of the tyre and the wheel centre, the kinematic rolling radius defined by motion and the kinetic rolling radius. His measurements were done at different tyre pressures on sandy loam soil. The reported measurements relied on the double integration of accelerometer measurements (to determine vertical wheel travel) and only the distance from the centre of the wheel to the road surface was considered as a dynamic radius. The kinematic rolling radius was defined as a function of slip and was defined with Equation (1) at different slip values. It is however unclear how the tyre slip was determined. The kinetic rolling radius was considered only under static conditions where the wheel's angular acceleration, the tyre dynamics and vibration modes were neglected and the longitudinal tyre force and the applied brake or driving torque are directly proportional, as shown in Equation (2). The kinetic rolling radius was reported as a function of inflation pressure.

$$
\begin{gathered}
r_{\text {kinematic }}=\text { distance travelled } / \text { total wheel rotation angle in radians } \\
\qquad M_{y}=F_{x} r_{\text {kinetic }}
\end{gathered}
$$

The aim of this paper is to investigate the rolling radius of a pneumatic tyre on both undulating and smooth hard roads and to look at the use of this rolling radius to determine longitudinal slip, specifically for application to ADAS systems intended for use on off-road vehicles such as SUVs. It was found that the overwhelming approach in the literature is to assume the rolling radius to be a constant parameter that can be determined under quasi-static conditions, but that there is little consensus among researchers as to how to define the rolling radius. Seldom is a distinction made between a kinematic rolling radius, used to relate the kinematics of a rolling wheel, and a kinetic rolling radius, which is used to relate the kinetics of torques applied to a wheel and the resulting longitudinal forces.

\subsection{KinEMATIC ROLLING RADIUS}

Before commencing with a detailed discussion on a pneumatic tyre's rolling radius, it is important to define kinematics as opposed to kinetics. Kinematics is the branch of dynamics that describes the motion of particles or bodies by referring only to the displacements, velocities and accelerations of those particles or bodies. Kinetics on the other hand is the study of the relations between unbalanced forces and moments and the resulting changes in motion (Meriam and Kraige, 2012).

The kinematic rolling radius of a tyre is used during the parameterisation and the simulation stages of longitudinal tyre modelling. The longitudinal force generating properties of a tyre is usually parameterised as a function of longitudinal tyre slip (Gillespie, 1992). The longitudinal tyre slip is calculated with Equation (3): 


$$
\begin{aligned}
& \lambda=\frac{\left|v-v_{c p}\right|}{v} \times 100 \% \\
& v_{c p}=\dot{\theta} \times r_{\text {kinematic }} \\
& \therefore r_{\text {kinematic }}=v_{c p} / \dot{\theta}
\end{aligned}
$$

\subsubsection{DEFINITION OF ZERO CONDITIONS FOR KINEMATIC ROLLING RADIUS}

The kinematic rolling radius in Equation (4) is assumed to be constant (the rigid disc assumption) and is defined under specified zero conditions. Two of the most common zero conditions are (Upadhyaya et al., 1988):

a) when there is a driving torque applied to propel the vehicle across a surface while delivering zero net traction, and

b) when the vehicle is pushed with zero axle torque. This is also the condition under which the Society of Automotive Engineers (SAE) defines the effective radius (Miller et al., 2001).

In both of these cases the kinematic rolling radius is defined with Equation (1) (Upadhyaya et al., 1988).

\subsubsection{PROBLEMS WITH THE DEFINITION OF THE KINEMATIC ROLLING RADIUS}

The problem with kinematic rolling radius under quasi-static conditions, such as those described above, is that the tyre deforms when driving. This deformation is due to numerous factors, such as rough roads, load transfer during vehicle manoeuvres, wear and excitation of the wheel hop frequency. Adcox et al. (2012) investigated the interaction between the tyre torsional dynamics and ABS control algorithms and found a significant increase in the stopping distance when the torsional dynamics were excited. It is possible that the reason for the increase in stopping distance is attributable to the definition of the rolling radius used to determine contact patch slip.

\subsubsection{CHALLENGES WITH MEASUREMENT OF THE PARAMETERS AND IMPLEMENTATION}

As shown in Equation (3), calculation of the longitudinal slip requires information of the vehicle speed (also known as the reference velocity) and the contact patch velocity. It is relatively easy to measure the reference velocity if a Global Positioning System (GPS) is fitted to the vehicle. A GPS is often not available on a commercial vehicle and typically has inadequate accuracy and sampling frequency and hence the reference velocity is estimated from measured wheel speeds. Penny and Els (2016) discussed the use of an algorithm utilising the measured wheel speed and static rolling radius to estimate reference velocity.

The measurement of the contact patch velocity is even more complicated than estimating the reference velocity. To estimate the contact patch velocity, it is usually assumed that the wheel is a rigid body. The kinematic relationship given in Equation (4) is then used to calculate the contact patch velocity (Meriam and Kraige, 2012). 
Measurement of the wheel speed $(\dot{\theta})$ is usually done with proximity switches and a pulse ring - this technique is universally implemented on commercial vehicles for ABS wheel speed measurement (Bosch, 2005).

The torsional dynamics of the tyre may contribute to a significant error when assuming the wheel is a single rigid body and measuring wheel speed on the wheel hub (Botha et al., 2015). This is especially true for soft, high aspect ratio tyres often used on off-road vehicles with coarse tread patterns and low inflation pressures. An alternative method for measuring the longitudinal slip of a tyre directly, using Digital Image Correlation (DIC), was proposed by Botha and Els (2014).

Care must also be taken when choosing a wheel speed filtering approach (Adcox et al., 2012, Penny and Els, 2016), especially on rough terrains. Delays in the wheel speed measurement may significantly influence the performance of such systems. Sensor vibration on rough terrains may also have a detrimental effect on the wheel speed measurement and control system designers are encouraged to ensure the rigidity of the sensor and elimination of its resonant frequencies. Errors may also be introduced when the tyres are used at different inflation pressures, different tyre brands are used or when there is significant tread wear present on the tyres.

\subsection{Kinetic ROLLING RADIUS}

The kinetic rolling radius is used to convert applied torque (either through applying friction brakes or torque from the drive shaft) to an effective longitudinal force, Figure 1 shows a free body diagram of the forces and moments acting on a wheel with a pneumatic tyre (from Equation (6)). The moment induced by the vertical force acting an offset distance from the geometric centre of the wheel is assumed to be the rolling resistance, as defined by Gillespie (1992). Further rolling resistance effects may be attributed to rubber hysteresis effects, but these are all assumed to be lumped into the $F_{z} \rho$ term. Also indicated in Figure 1 are the angular velocity and wheel centre velocity of Equations (3) and (4). By considering the wheel rotating about the axle, the system reduces to planar motion and Euler's extension of Newton's Second Law from particles to rigid bodies may be used (Meriam and Kraige, 2012):

$$
\begin{gathered}
\sum M_{O}=I_{O} \ddot{\theta} \\
F_{x} r_{\text {kinetic }}-F_{z} \rho-M_{y}=I_{O} \ddot{\theta} \\
\therefore r_{\text {kinetic }}=\frac{I_{O} \ddot{\theta}+F_{z} \rho+M_{y}}{F_{x}}
\end{gathered}
$$

This rolling radius is thus a kinetic relationship and not the same as the kinematic rolling radius used in Equation (4).

The aim of this study is to investigate the kinematic and kinetic rolling radius of a pneumatic tyre on both undulating and smooth hard roads and to evaluate the validity of common assumptions discussed above. 


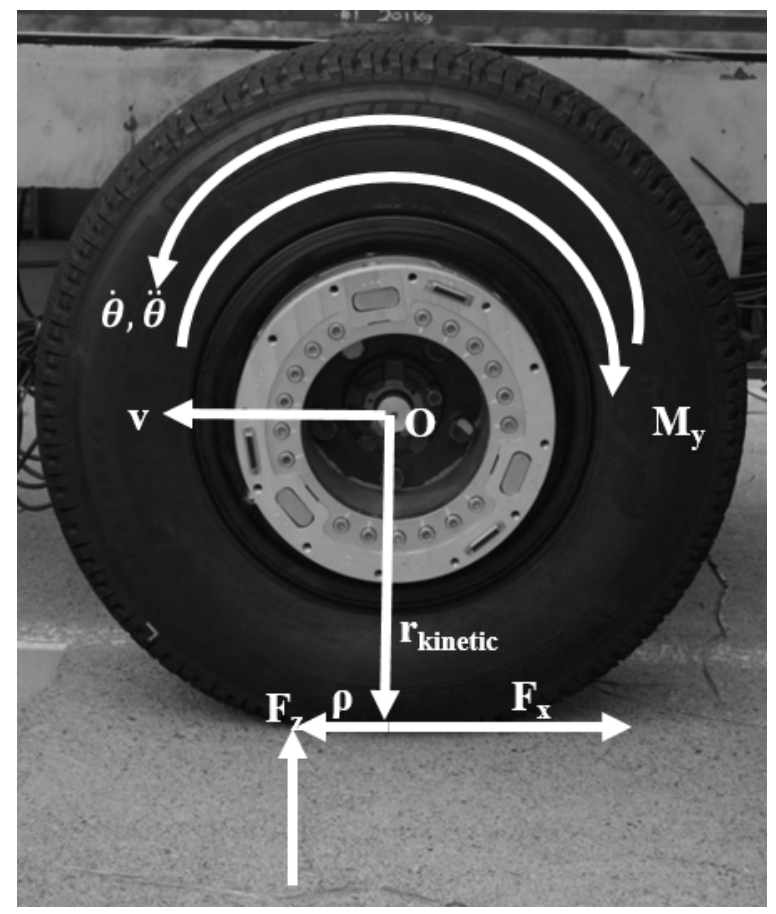

\section{FIGURE 1 - FORCES AND MOMENTS ACTING ON A BRAKED TYRE}

The first step in the investigation is to compare the kinematic and the kinetic rolling radius. The following research questions are investigated:

a) How does the dynamic kinematic rolling radius measured experimentally compare with the static kinematic rolling radius defined under the abovementioned zero-conditions?

b) Is it valid to use a static or quasi-static definition of the rolling radius to determine contact patch velocity?

c) Can the wheel be approximated as a rigid body so that the kinetic rolling radius can be used to convert the applied brake torque to the applied brake force?

An experimental setup that uses a wheel force transducer to measure the applied forces and moments (to determine the kinetic rolling radius) and a dual-camera vision-based measurement system that measures the contact patch velocity (to determine the kinematic rolling radius) was developed in an attempt to answer these research questions. The tests were conducted on a SUV braking on a smooth paved road and on a Belgian Paving. The experimental results thus include the effects that would be experienced by the tyre in a real world situation and not laboratory conditions.

\section{EXPERIMENTAL SETUP}

From Equations (3), (4) and (6) it is evident that the following states need to be measured in order to determine the two roll radii of interest:

a) Wheel angular velocity and angular acceleration

b) Vehicle velocity

c) The forces and moments acting on the wheel 
In addition to the above mentioned states, the mass and inertia parameters are also required. The tyre used for this study is a Michelin LTX AT ${ }^{2}$ 235/85/R16 that is typically fitted to SUVs with a measured mass of $19 \mathrm{~kg}$ and inertia about the spin axis of $3.1 \mathrm{~kg} \cdot \mathrm{m}^{2}$. All the tests were performed at $200 \mathrm{kPa}$ tyre pressure on the front left tyre of the vehicle. Data was recorded while the vehicle was braked from approximately $45 \mathrm{~km} / \mathrm{h}$ on both a smooth road and a Belgian Paving test track. The tests were repeated several times to determine the repeatability of the tests. Data acquisitioning was performed on a Helios Single Board Computer (from this point on referred to simply as the Data Acquisitioning System or DAQ) at a sampling rate of $1000 \mathrm{~Hz}$. The experimental setup is shown in Figure 2.

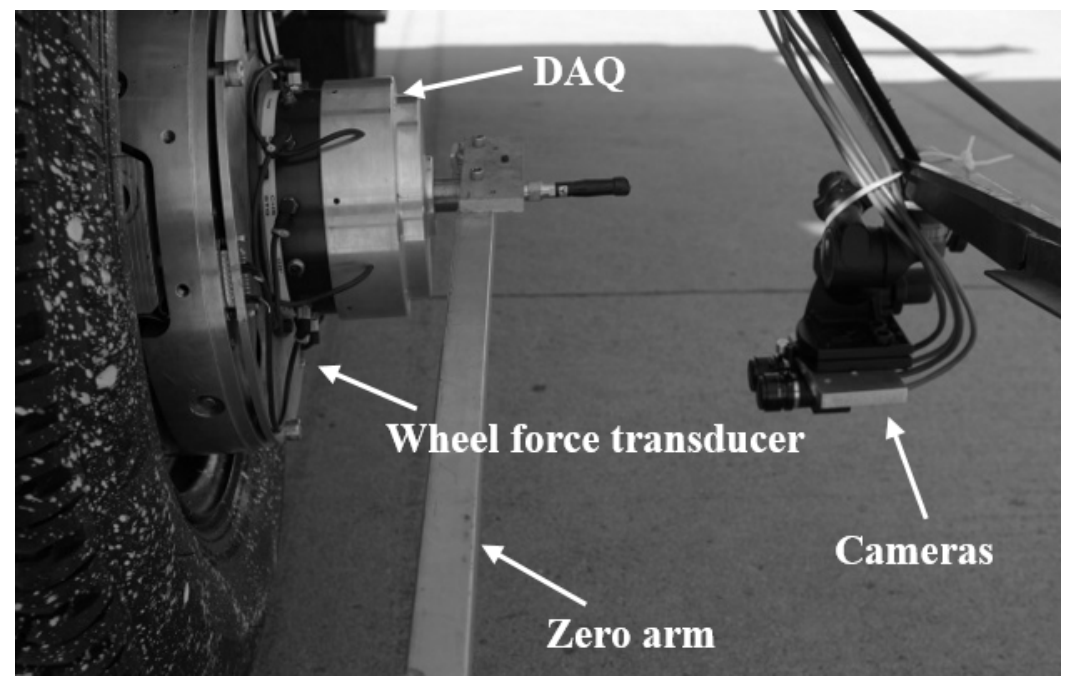

FIGURE 2 - EXPERIMENTAL SETUP

To compare the kinematic rolling radius of definition (b) of Section 1.1.1 with the kinematic rolling radius experienced during a braking test, Equation (4) is rearranged as shown in Equation (7):

$$
r_{\text {kinematic }}=\frac{v_{c p}}{\dot{\theta}}
$$

From Equation (7) it is evident that contact patch velocity and wheel angular velocity are to be measured to determine the kinematic rolling radius. Wheel speed is measured with inductive proximity switches. Sixty (60) indentations have been machined in the brake disc. When the wheel rotates, a square wave related to the angular velocity of the wheel is generated and converted to a voltage with a frequency to voltage converter. A disadvantage of this measurement approach to angular velocity is possible poor performance at lower angular velocities and high angular acceleration (e.g. at wheel lockup). The frequency to voltage converter may also introduce a slight delay in the measurement. Vehicle speed was measured with a NovAtel SPAN-CPT Single Enclosure GNSS/INS Receiver. The SPAN-CPT makes use of both the GPS and GLONASS systems.

The contact patch velocity, $v_{c p}$, is measured directly using Digital Image Correlation (DIC) (Botha and Els, 2014). DIC is a mathematical measurement technique used to track changes in an image. There are several algorithms that can be used, but the basic approach remains the 
same. Regions in successive images are matched, effectively tracking these regions. These regions are often simplified to several key points. Initially key points are identified using their uniqueness and ease of tracking from one image to the next. After identifying these key points they are matched from one image to the next. There are several feature descriptors in the literature, the Scale-Invariant Feature Transform (SIFT) feature descriptor is used in this study (Botha and Els, 2014, Lowe, 2004). The SIFT algorithm finds unique points in an image as well as identifies these key points using a feature vector which describes local gradients around each key point. These feature vectors are used to identify the key points in different images and therefore allows them to be tracked across images, as shown in Figure 3. A time sequence of images is then recorded at a predefined frequency. Thus, if points are tracked across consecutive images the velocity of these points can also be determined.

A calibrated two camera setup was mounted on the outside of the left front wheel of the test vehicle (see Figure 2). The two camera system is capable of measuring 3D surfaces. However, this requires textured surfaces to be able to match features between the two different camera views. The road surface normally contains sufficient texture, but the tyre normally has a monotonous colour and thus few features to track. Texture was added by spraying the sidewall of the tyre with white paint or using a white marker to make arbitrary marks or spots as can be seen in Figure 3.

Points are tracked over an area encompassing both the tyre and road. The interface between the tyre and road are first determined. Points which lie below this interface are classified into point on the road. Points above and close to the interface are classified into tyre points. A RANSAC algorithm (Fischler and Bolles, 1981) is used to determine the average velocities of the tyre and road using all points, while removing outliers.

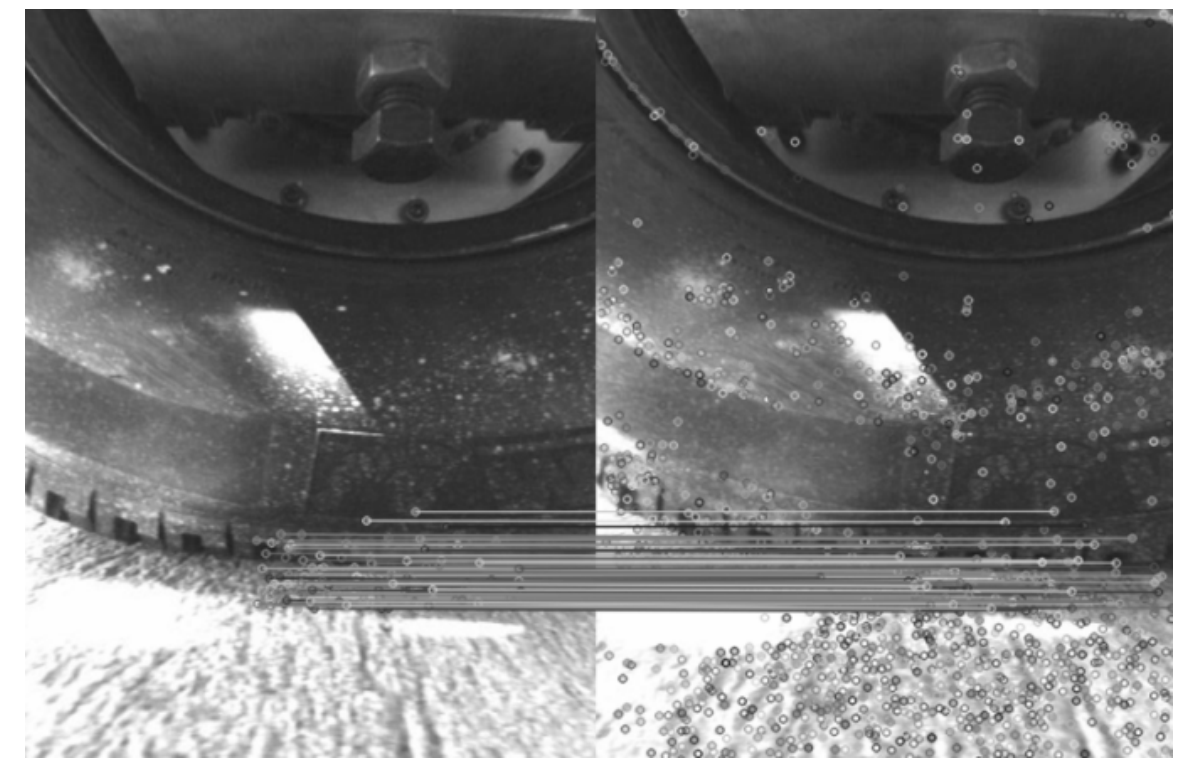

\section{FIGURE 3 - TRACKING KEY POINTS IN THE CONTACT PATCH ACROSS TWO} CONSECUTIVE FRAMES

This allows for the linear velocity of the tyre at the contact patch and vehicle speed to be measured directly, thus enabling the rolling radius to be determined from Equation (7). The 
camera frames are synchronised with the other captured data using trigger pulses. A frame is taken at half the frequency of the other captured data (due to the physical limitations of the cameras). This allows the camera data to be easily synchronised with other measurements.

Figure 4 shows the result of the classification algorithm applied to the DIC data in $2 \mathrm{D}$ format. A 3D format image containing the clustering results is shown in Figure 5. Points classified as lying on the tyre are indicated as green and points on the road are blue. The remaining points are classified as outliers and are magenta and cyan. This shows that the algorithm classifies the points correctly with no errors in either the tyre or road set.

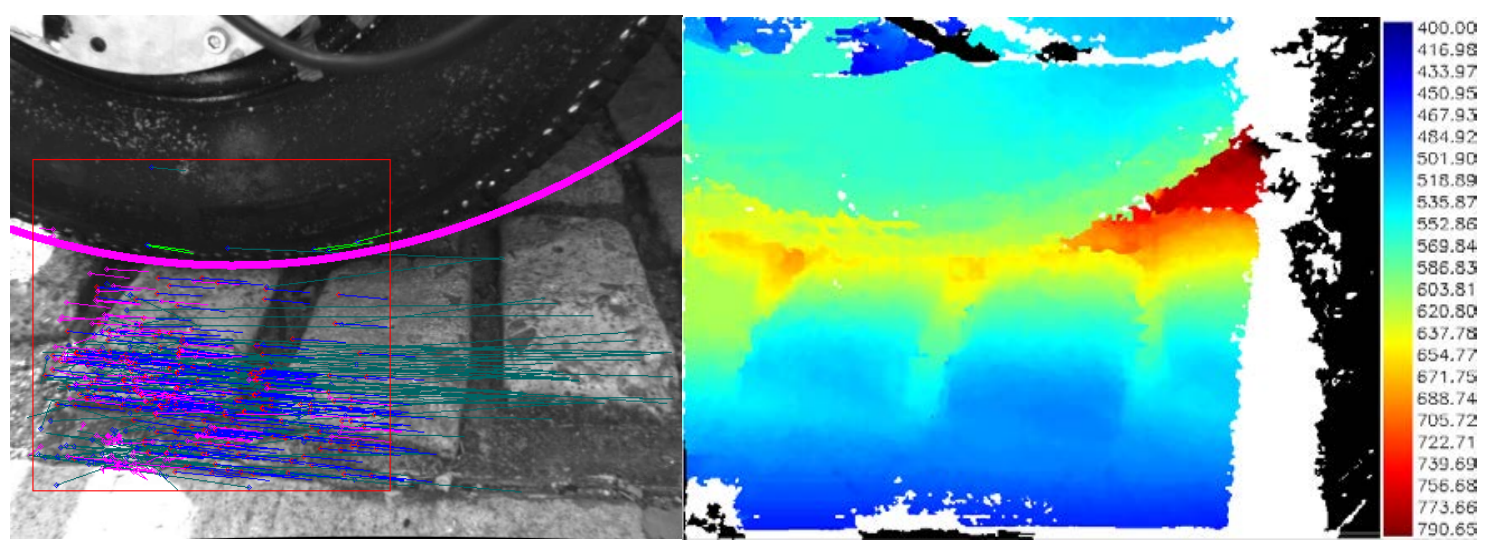

FIGURE 4 - CLUSTERING POINTS INTO GROUND AND OUTLIER POINTS IN THE 2D IMAGE

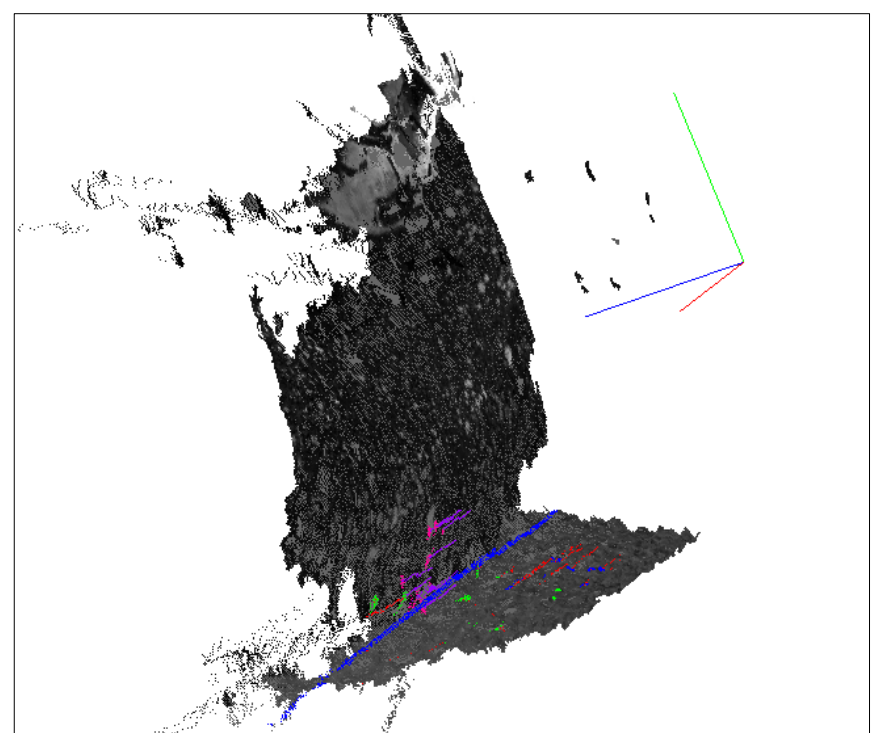

\section{FIGURE 5 - CLUSTERING OF POINTS INTO TYRE, GROUND AND OUTLIER POINTS IN A 3D POINT CLOUD}

To calculate the kinetic rolling radius used in the force balance in Equation (6), the wheel forces and moments shown in Figure 1 need to be measured along with the wheel's angular acceleration. A wheel force transducer (WFT) developed by Becker and Els (2012) was used to measure the forces and moments acting on the wheel. The WFT consists of six single component load cells arranged in the rim of the wheel. The wheel's rotation angle is measured 
with an encoder that is held stationary with a zero arm, attached to the vehicle axle. The wheel rotation angle is used to convert the forces measured by the single component load cells to forces and moments oriented along the tyre coordinate system. The static wheel load was approximately $650 \mathrm{~kg}$. The measured wheel angular velocity's time derivative is used to calculate wheel angular acceleration.

The WFT data is captured on a DAQ that is mounted to the WFT. Two DAQs are thus used, one in the vehicle that measures wheel and vehicle speed and the other mounted to the WFT. A trigger pulse is generated by the DAQ on the vehicle. The trigger pulse is used to synchronise the data sets captured on the two DAQs.

\section{EXPERIMENTAL RESULTS}

The experimental results for both the kinematic and kinetic roll radii are now discussed.

\subsection{KINEMATIC ROLLING RADIUS}

The zero definition of Section 1.1.1 (b) is used to determine the kinematic rolling radius under static conditions. This kinematic rolling radius is used as the benchmark for comparative purposes. A mark is made on the ground directly below the wheel centre on the road and on the tyre and the vehicle is pushed until the wheel has made a full rotation and another mark is made on the road. The distance between the two marks is then measured and the kinematic rolling radius defined by Equation (1) is calculated. This benchmark kinematic rolling radius was found to be $0.3875 \mathrm{~m}$.

The kinematic rolling radius is calculated with Equation (7) and the results for braking on a smooth road from $12 \mathrm{~m} / \mathrm{s}$ are shown in Figure 6. In Figure 6(a) the vehicle speed as measured with a GPS is compared to the speed as measured with the cameras and a close correlation is evident. The DIC technique therefore accurately measures road speed. Figure 6(b) shows the kinematic rolling radius as calculated with Equation (7) from the DIC data.

Figure 7 indicates the kinematic rolling radius while braking on the Belgian Paving. From Figure 6 and Figure 7 it may be seen that the rolling radius reduces towards the end of the test run and eventually becomes zero, which is an unrealistic value. Some of the initial reduction in rolling radius may be due to load transfer during braking. Further reduction in the rolling radius is a numerical issue. This is due to Equation (7), where a small contact patch velocity speed will reduce the result of Equation (7) to zero. At these low speeds, a small unaccounted delay error in the frequency to voltage converter may also significantly influence the results. 
(a) Vehicle speed

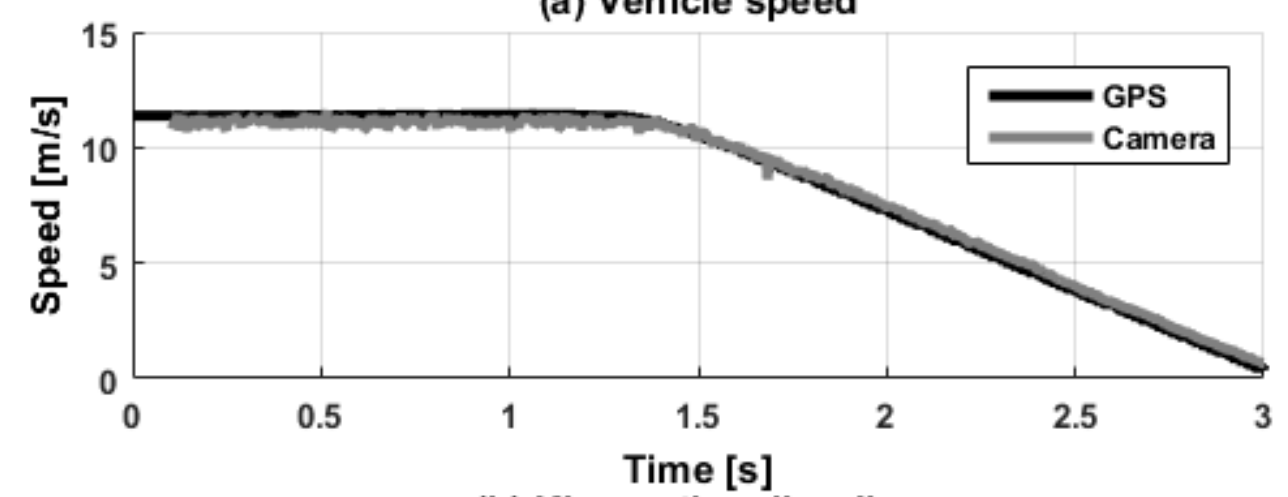

(b) Kinematic roll radius

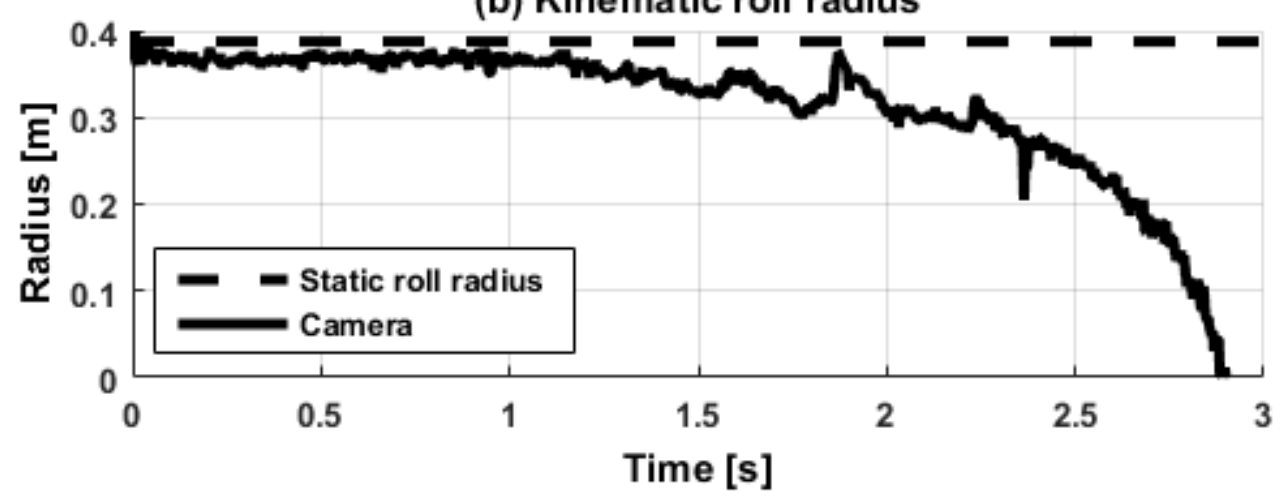

FIGURE 6 - (A) VEHICLE SPEED COMPARISON AS MEASURED WITH A GPS AND THE DIC TECHNIQUE AND (B) THE CALCULATED KINEMATIC ROLLING RADIUS ON A SMOOTH ROAD

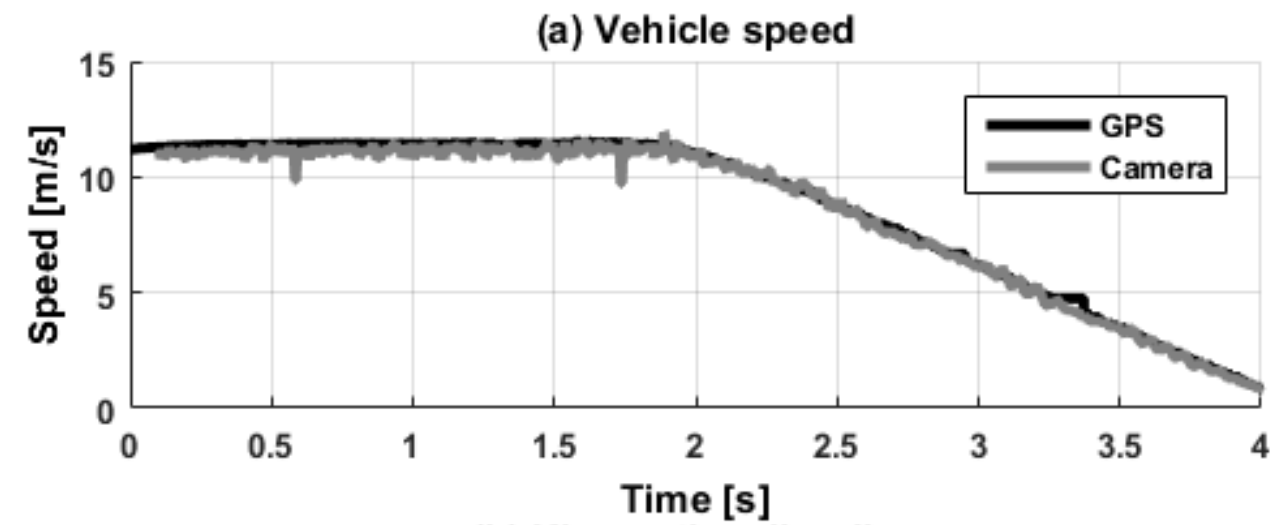

(b) Kinematic roll radius

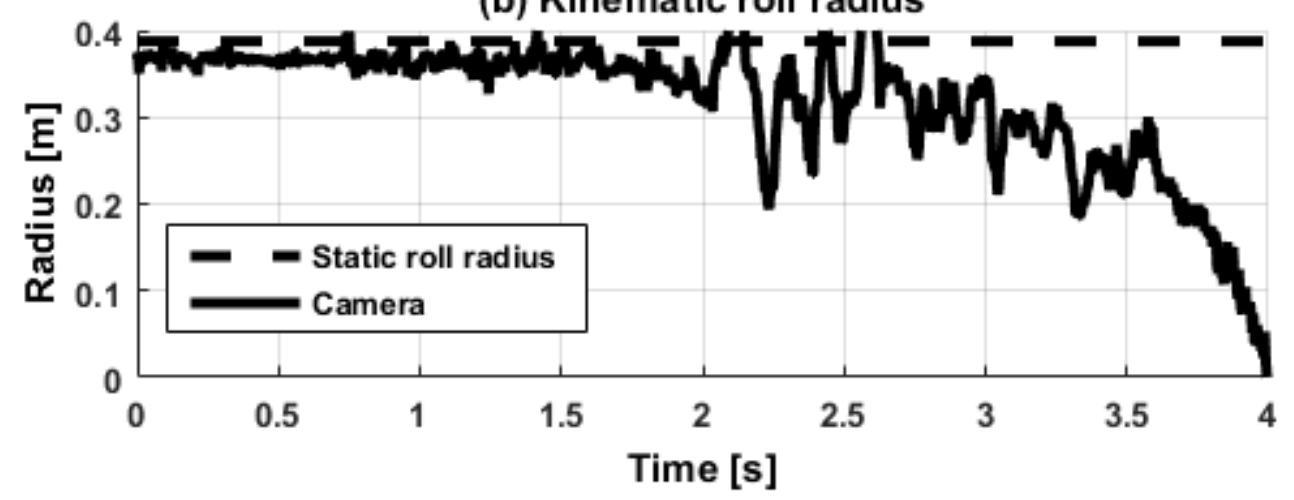

FIGURE 7 - KINEMATIC ROLLING RADIUS ON BELGIAN PAVING 
Rather than comparing the kinematic rolling radius as shown in Figure 6 and Figure 7, Figure 8 compares the contact patch velocity calculated with Equation (4). As stated in the Introduction, Equation (4) relies on assuming the wheel to be a rigid body. The static kinematic rolling radius was measured using definition b) in Section 1.1 .1 and found to be $0.3875 \mathrm{~m}$. Figure 8 indicates that the contact patch velocity, predicted by Equation (4), is a good approximation when compared to the velocity measured with DIC. Defining the rolling radius with definition b) in Section 1.1.1 gives an accurate and smooth result. On the Belgian Paving (shown in Figure 9), however, a significant variation is observed between the contact patch velocity as estimated with Equation (4) and the DIC measured contact patch velocity. There are significant differences between the DIC measured contact patch velocity and that of Equation (4) when looking at the peaks between 2 and 3 seconds in Figure 9. This may filtering effect may be due to the filtering of the wheel speed signal and may account for the poor performance of ADAS systems on rough roads.

An example of the error made by using definition b) in Section 1.1.1 to define the kinematic rolling radius is clearly visible in Figure 8 and Figure 9. During the first portion of both figures, before braking commences, the contact patch velocity calculated with Equation (4) gives a higher speed than the DIC measured contact patch velocity. Since Equation (4) is a linear relationship, the error margin will increase at higher speeds and significant errors may be made. Using definition b) in Section 1.1 to define the kinematic rolling radius does not take tyre wear, different inflation pressures and the weight transfer of the vehicle into account.

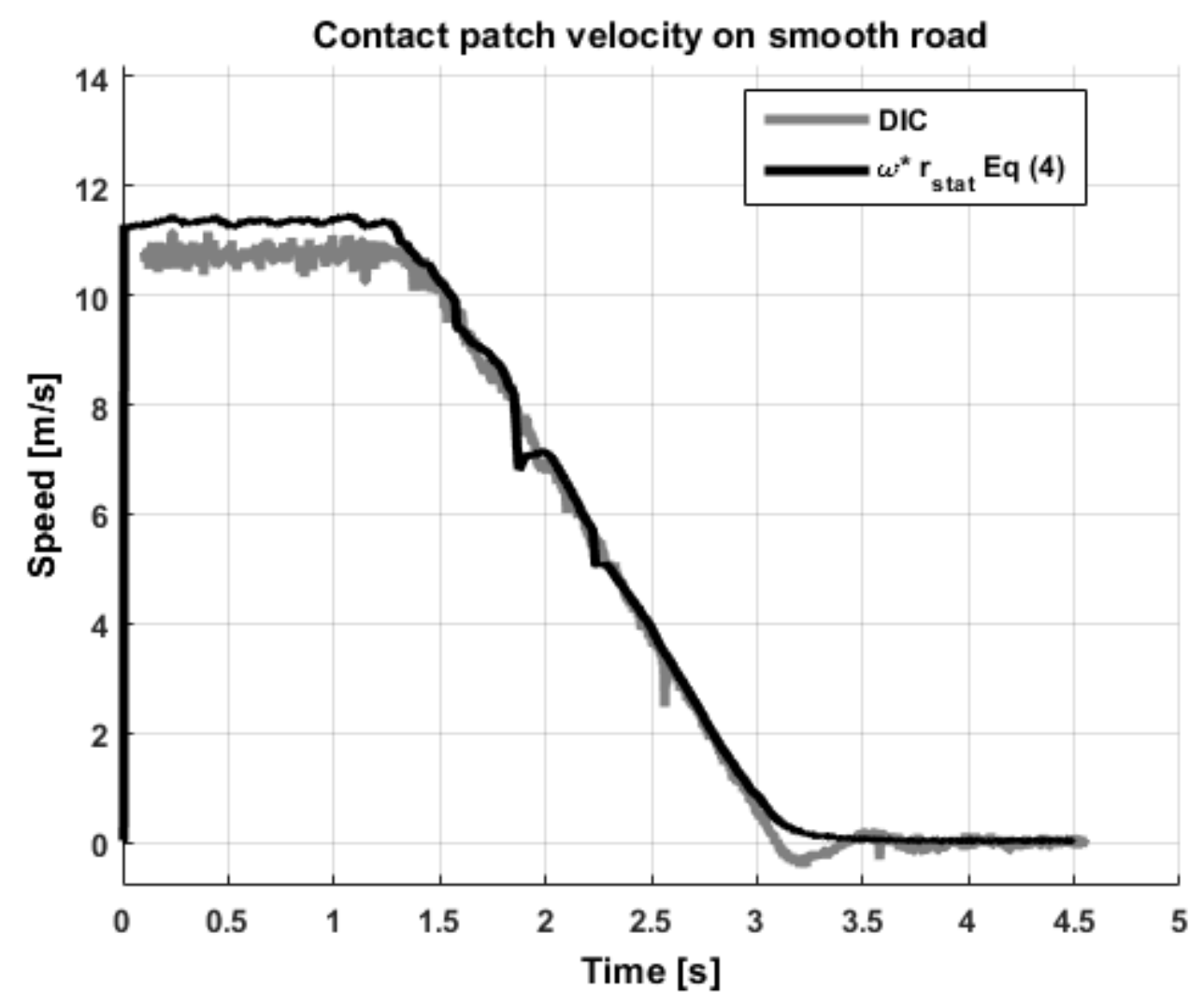

FIGURE 8 - CONTACT PATCH VELOCITY ON A SMOOTH ROAD 


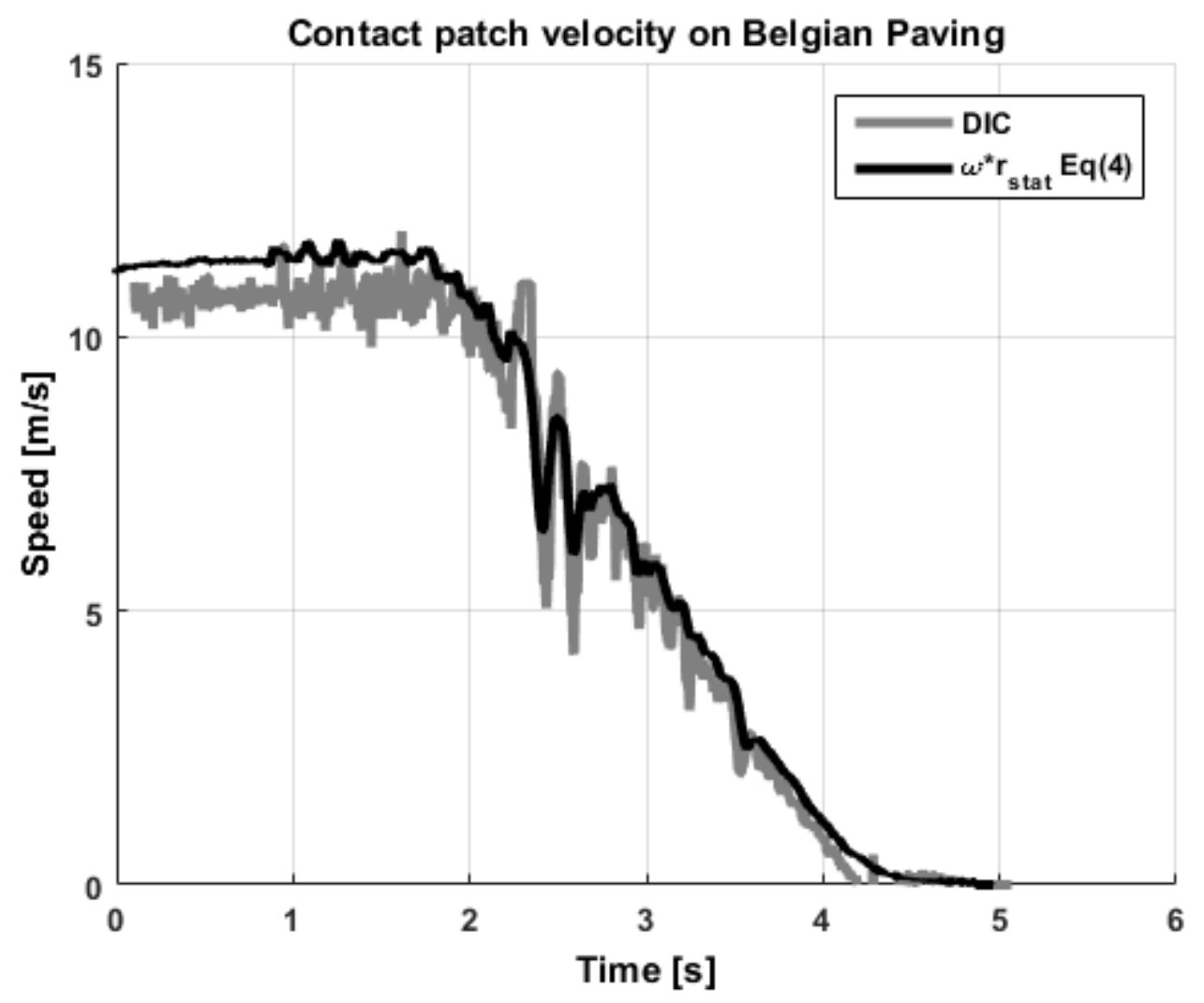

FIGURE 9 - CONTACT PATCH VELOCITY ON BELGIAN PAVING

\subsection{KINETIC ROLLING RADIUS}

Figure 10 shows the resulting brake force and moment and the corresponding vehicle speed for a test run on the Belgian Paving. Initially, for the first second, the variation in vertical force is small. The variation then suddenly increases as the vehicle drives from the run-up area, which is smooth concrete, onto the undulating Belgian Paving. Instants when ground contact is lost may be seen when the vertical force reduces to zero. At approximately two seconds, the brake torque is increased and a corresponding increase in the longitudinal force and brake torque can be seen. At this stage the vehicle speed starts to decrease.

The kinetic rolling radius of the wheel was calculated by rearranging Equation (7) as:

$$
r_{\text {kinetic }}=\frac{I_{o} \ddot{\theta}+F_{z} \rho+T_{y}}{F_{x}}
$$

The rolling resistance included in the $F_{z} \rho$ term was determined by measuring the moment about the wheel axle when performing a coast-down test while the vehicle was not in gear. The coast-down tests were performed on both the smooth road and on the Belgian Paving and the root mean square (RMS) of the rolling resistance moment was calculated. The RMS rolling resistance moment was included as the $F_{z} \rho$ term in Equation (8). Table 1 shows the RMS rolling resistance moments.

\section{TABLE 1 - RMS ROLLING RESISTANCE ON SMOOTH ROAD AND BELGIAN} PAVING 


\begin{tabular}{|l|l|}
\hline Road & RMS Rolling resistance [N.m] \\
\hline Smooth & 18.1 \\
\hline Belgian paving & 38.0 \\
\hline
\end{tabular}

The resulting kinetic rolling radius is shown in Figure 11 and it immediately becomes evident that the rigid disc (or rigid body) assumption allowing the use of Equation (8) is invalid. Bearing in mind that the tyre has dimensions 235/85/R16 (and thus a maximum unloaded diameter of $805.9 \mathrm{~mm}$ ), the kinetic rolling radius is not expected to exceed $403 \mathrm{~mm}$. The tyre is fitted to a 16 ” rim, which means the rim radius is at $0.203 \mathrm{~m}$ and rolling radius values below that indicates contact between the rim and the road surface and tyre damage should occur. This did not happen. Even on the smooth road, the kinetic rolling radius lies almost exclusively above 400mm. On the Belgian Paving Equation (7) becomes unstable since the measured brake force is sometimes very close to zero as the tyre traverses the cobblestones. Additional noise is added to the system because the angular acceleration of the wheel was not measured and $\ddot{\theta}$ has to be derived from the measured angular velocity. It is evident that the assumption of a rigid disc to approximate the forces acting on a wheel is invalid under these conditions.
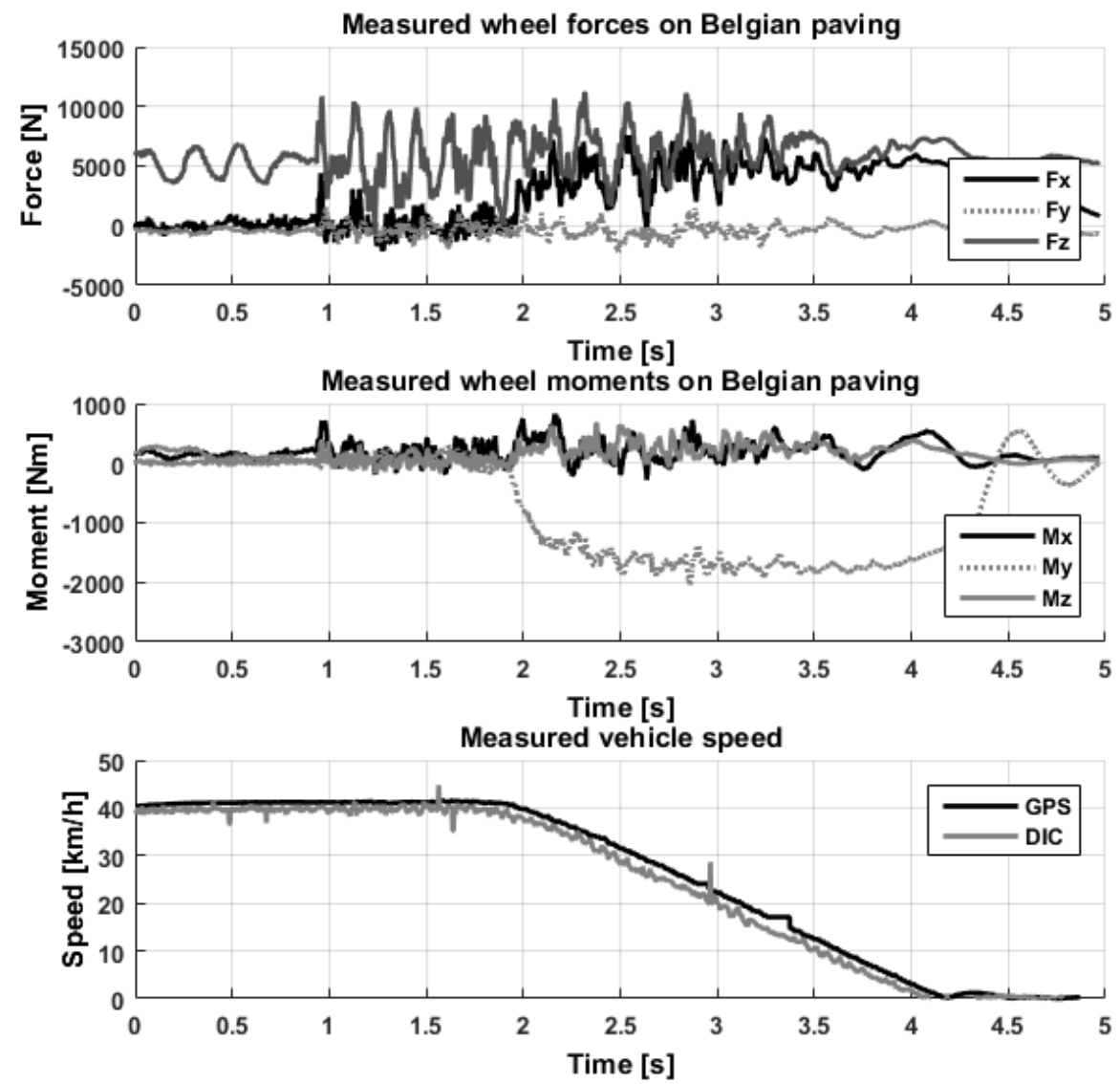

FIGURE 10 - MEASURED FORCES, MOMENTS AND VEHICLE SPEED ON BELGIAN PAVING 


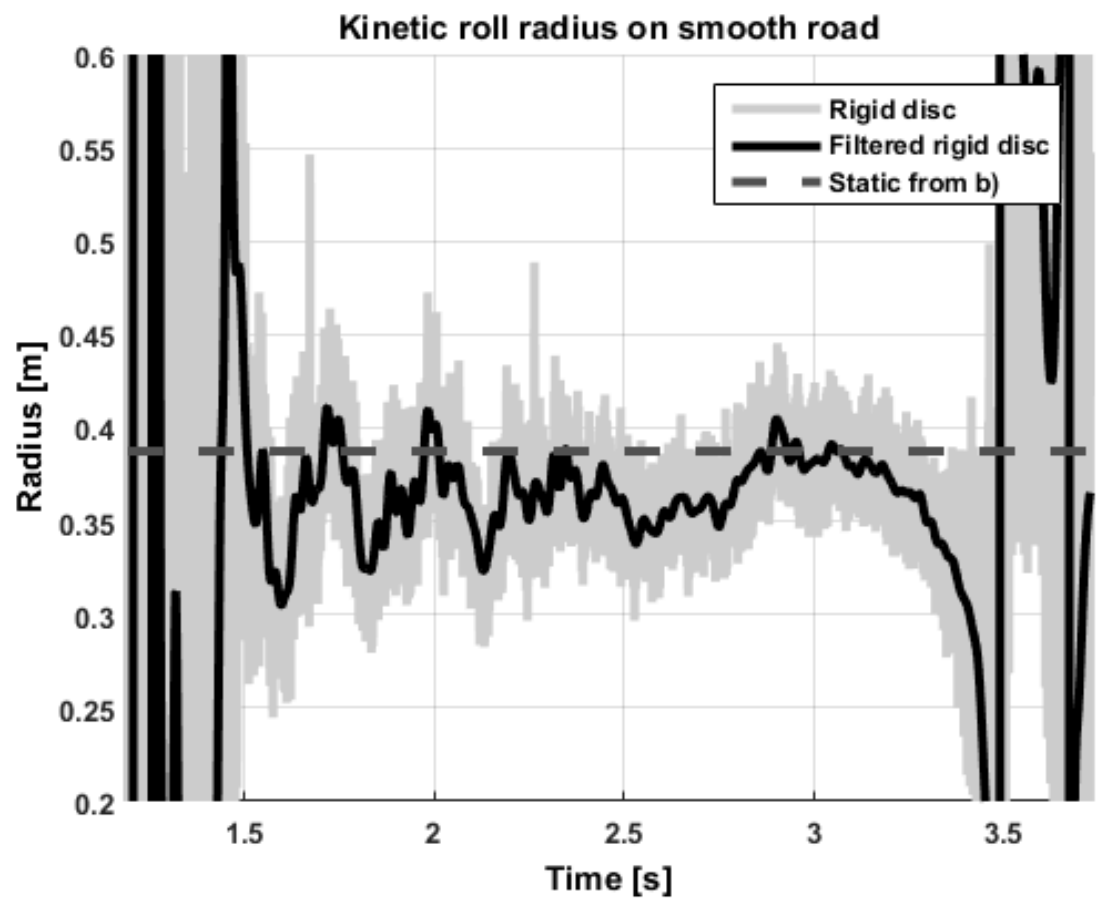

FIGURE 11 - RIGID BODY CALCULATION OF KINETIC ROLLING RADIUS ON SMOOTH ROAD (NOTE THAT ONLY THE BRAKING PORTION OF THE TEST RUN IS SHOWN)

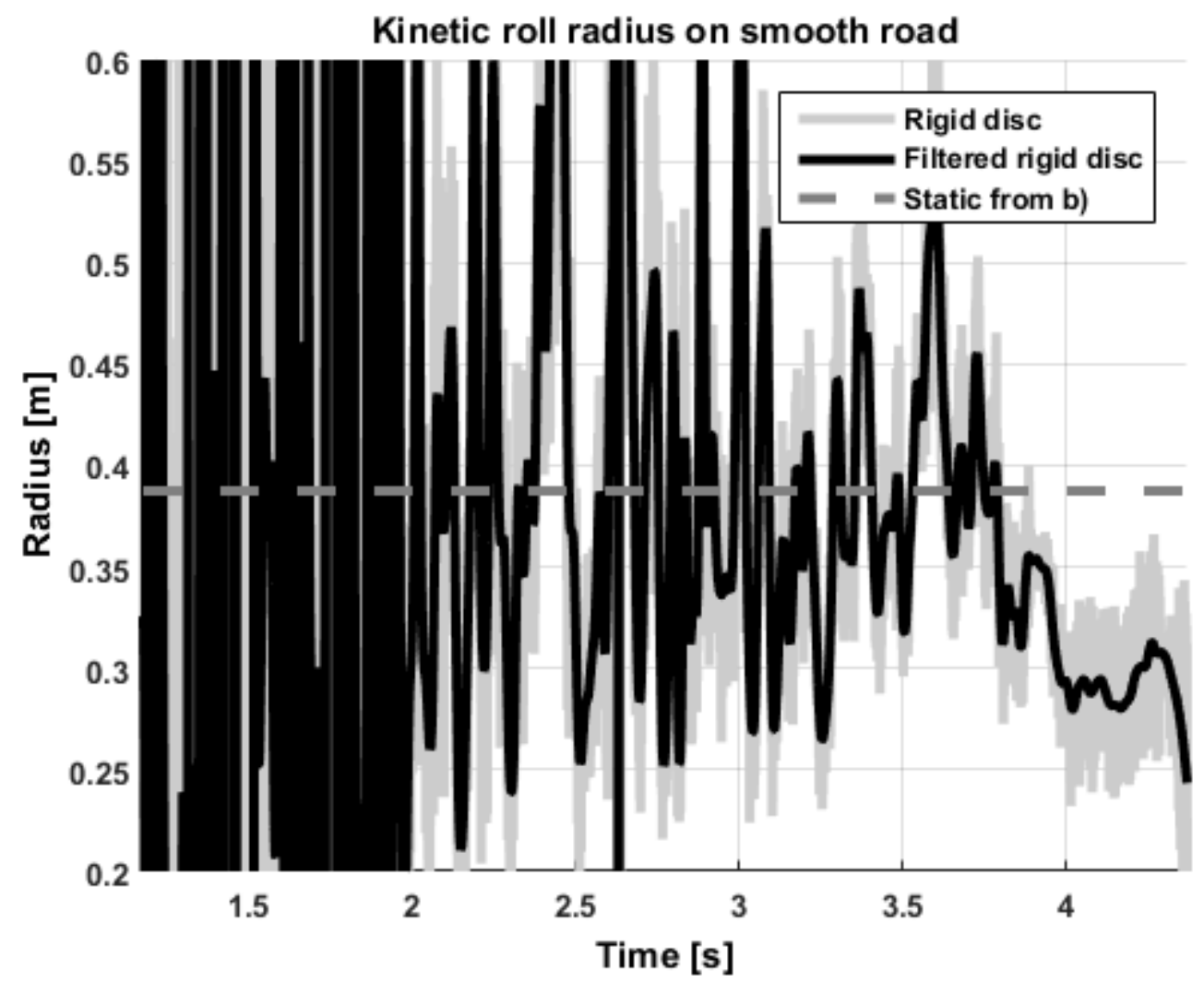

FIGURE 12 - RIGID BODY CALCULATION OF ROLLING RADIUS ON BELGIAN PAVING (NOTE THAT ONLY THE BRAKING PORTION OF THE TEST RUN IS SHOWN) 
To improve the kinetic rolling radius results, the measured forces, moments and wheel angular acceleration were zero-phase filtered with a $50 \mathrm{~Hz}$ low pass Butterworth filter. Zero-phase filtering processes the input data in both the forward and reverse directions, resulting in zerophase distortion. Butterworth filters have a magnitude response that is maximally flat in the passband and monotonic overall. A compromise between this smoothness and the roll-off steepness results in reduced roll-off steepness. $50 \mathrm{~Hz}$ was chosen as a cut-off frequency, because it is known that the tyre natural frequencies of interest lie below this cut-off frequency (wheel hop frequency is typically at about $15 \mathrm{~Hz}$ and the tyre torsional frequency is known to lie at $28.4 \mathrm{~Hz}$, measured by Bosch et al. (2016)). Filtering markedly improved the kinetic rolling radius estimate on the smooth road, but it had a negligible effect on the results on the Belgian Paving. The filtered results are also indicated in Figure 11 and Figure 12. The filtering still did not improve the kinetic rolling radius results sufficiently to justify the use of a rigid disc to model the tyre.

Figure 13 and Figure 14 show the Fast Fourier Transform (FFT) magnitudes for the vertical and longitudinal tyre force and brake moment on the smooth road and the Belgian Paving, respectively. When comparing the results shown in Figure 13 and Figure 14 it becomes evident that the frequency content excited by the Belgian Paving is significantly more than on the smooth road, as expected. A definite peak is seen in the vertical tyre force on the smooth road at approximately $4 \mathrm{~Hz}$. This corresponds to the pitch natural frequency of the vehicle and hence the load transfer between front and rear axles during braking. The same peak at $4 \mathrm{~Hz}$ is present in Figure 14, but an additional peak is seen at $5.6 \mathrm{~Hz}$. The peak at $5.6 \mathrm{~Hz}$ was evident on all the runs on the Belgian Paving, but not on the smooth road. It may thus be concluded that the $5.6 \mathrm{~Hz}$ peak was excited by the terrain input, but that the $4 \mathrm{~Hz}$ peak is independent of the terrain. In Figure 13 and Figure 14 a small peak may be seen at $16 \mathrm{~Hz}$, this corresponds to the wheel hop natural frequency. There is some evidence of a peak at around $35 \mathrm{~Hz}$ on the longitudinal tyre force FFT magnitudes. This corresponds with the tyre's torsional natural frequency and justifies the use of $50 \mathrm{~Hz}$ as a low-pass filtering cut-off frequency.

The dynamic behaviour of the tyre under dynamic conditions renders the rigid disk assumption invalid. This can lead to the underperformance of ADAS systems that rely on the constant rolling radius assumption.

\section{CONCLUSIONS AND FUTURE WORK}

The aim of this investigation was to determine the validity of using constant kinematic and kinetic roll radii of a pneumatic tyre on both undulating and smooth hard roads. The overwhelming approach in the literature is to assume the rolling radius to be a constant parameter that can be determined under quasi-static conditions. No distinction is made between a kinematic rolling radius, used to relate the kinematics of a rolling wheel, and a kinetic rolling radius, which is used to relate the kinetics of torques applied to a wheel and the resulting longitudinal forces. To answer the research questions posed in Section 1.2: 

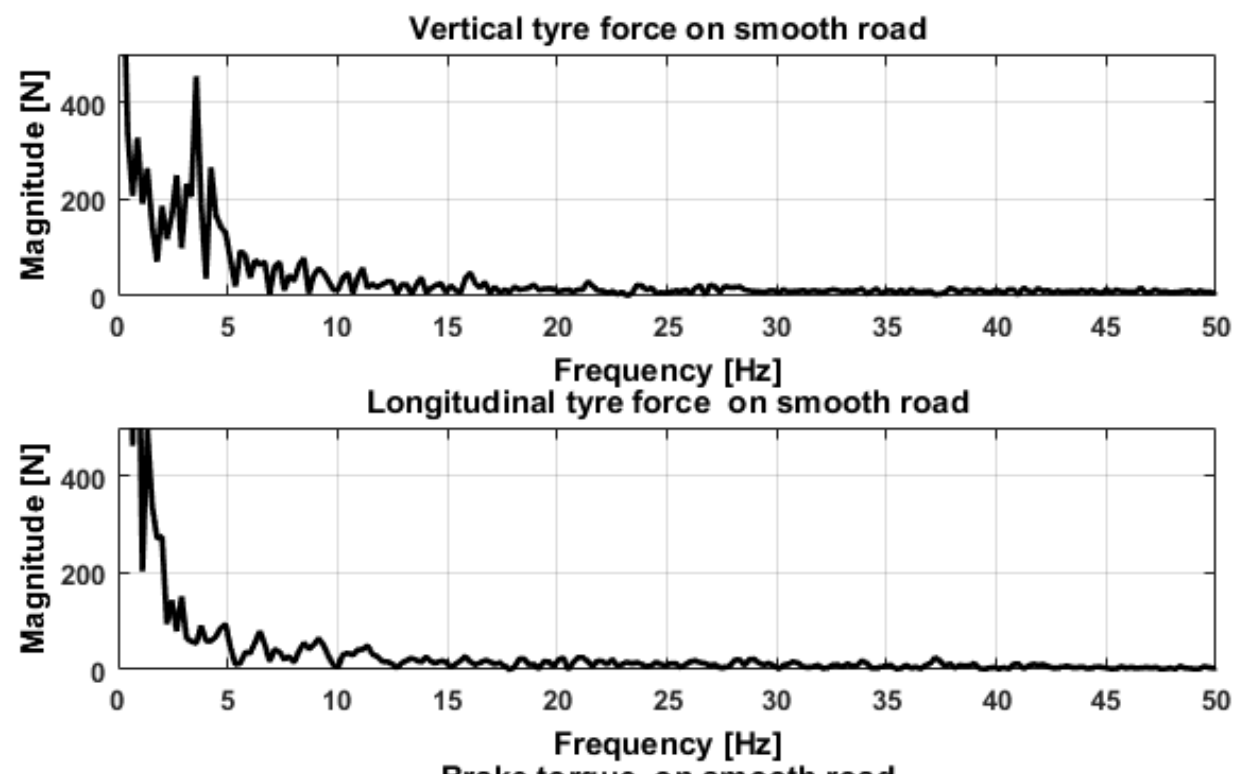

Brake torque on smooth road

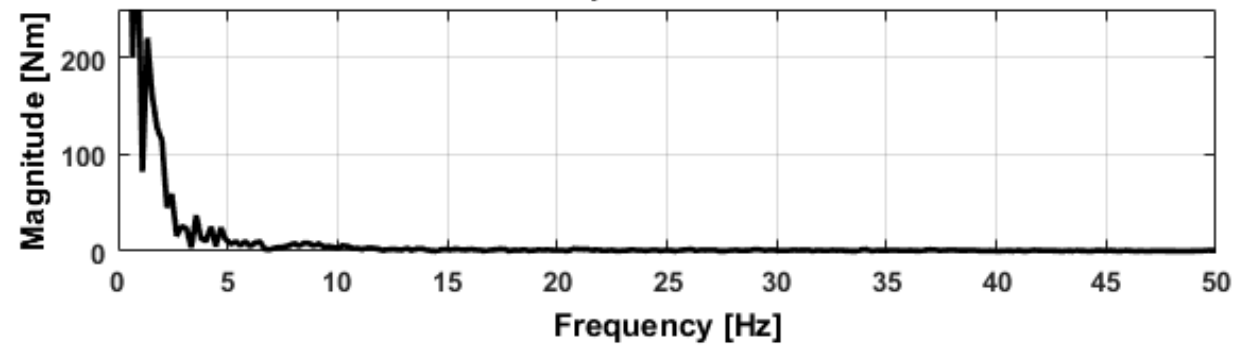

FIGURE 13 - FFT MAGNITUDES OF MEASURED FORCES ON SMOOTH ROAD
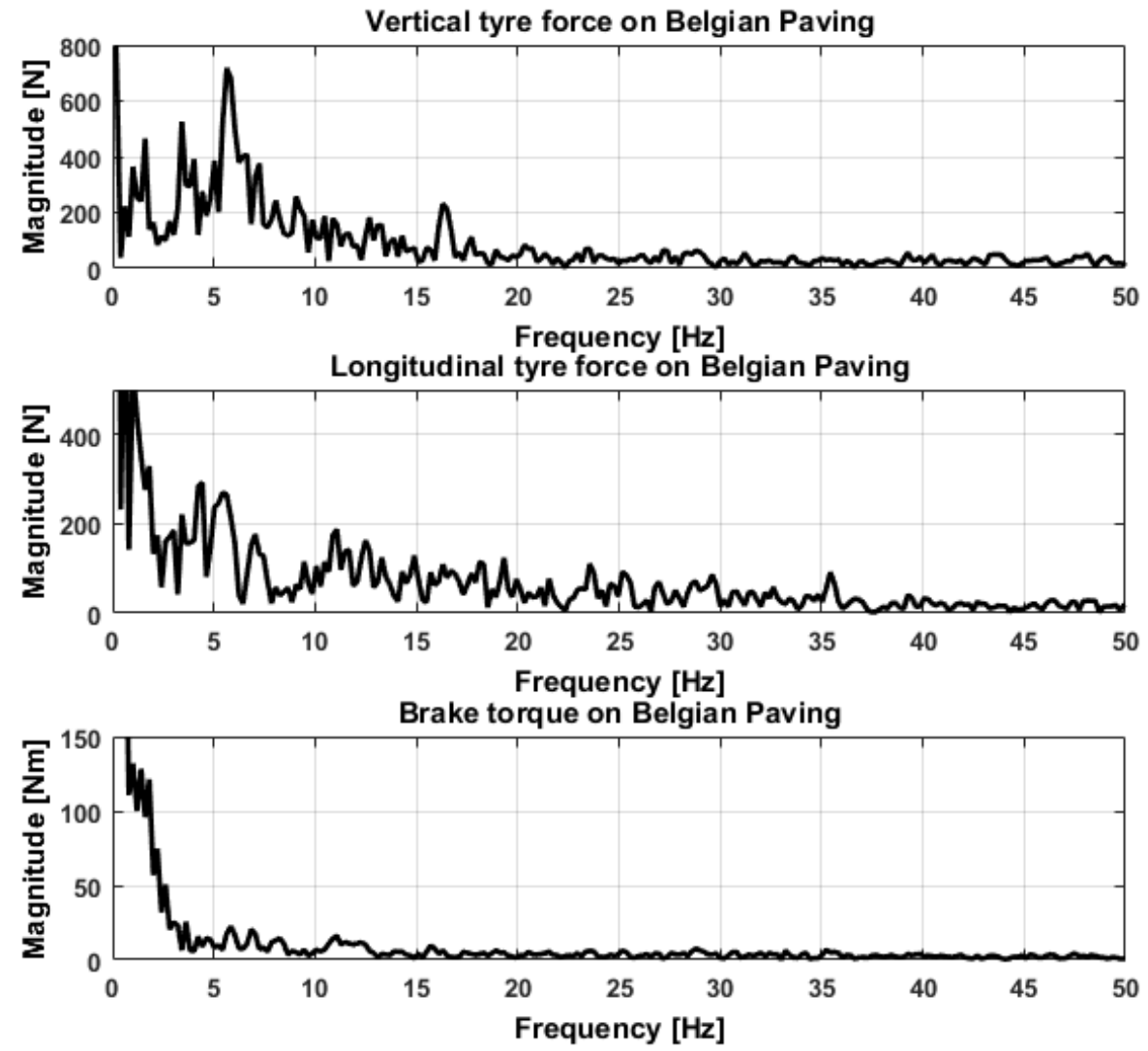


\section{FIGURE 14 - FFT MAGNITUDES OF MEASURED FORCES ON BELGIAN PAVING}

a) How does the dynamic kinematic rolling radius measured experimentally compare with the static kinematic rolling radius defined under the abovementioned zero-conditions? Is it safe to use a static or quasi-static definition of the rolling radius to determine contact patch velocity?

The rolling radius defined by Equation (1), under the condition of zero applied torque, is a valid assumption when used on a smooth, hard road surface. It may be used to estimate contact patch velocity and thus longitudinal slip under these conditions. This conclusion is supported by the success of modern driver assist systems, such as traction control and ABS, when driving on smooth hard roads.

The rolling radius defined by Equation (1) gives acceptable results when used to estimate contact patch velocity on hard, undulating surfaces such as a Belgian Paving. There are some differences in the peaks seen in Figure 9 that may contribute to the poor performance of ADAS systems on rough roads such as the Belgian Paving. Some signal conditioning and filtering may be necessary for the wheel speed measurement that may account for the differences in the peak values.

It must however be noted that the contact patch velocity determined with the static rolling radius gives acceptable results on the rough road, and thus does not explain why ABS algorithms' performance deteriorates when active on a rough road. The reduction in performance of these systems must thus also be attributed to other physical phenomena, probably associated with the tyre transient behaviour. Another possible explanation is given by Adcox et al. (2012) . Their investigation looked at the filtering (specifically the cut-off frequency of a low pass filter) of the wheel speed signal that is used to determine the wheel's angular acceleration. ABS algorithms often use both longitudinal slip thresholds and angular acceleration thresholds to modulate brake pressure. It is possible that the noise induced by deriving angular acceleration form wheel speed and the filtering of the wheel speed that is required has a larger influence on the ABS algorithm's performance than originally expected. Further research on this topic is required.

b) Can the wheel be approximated as a rigid body so that the kinetic rolling radius can be used to convert the applied brake torque to the applied brake force?

The assumption that the wheel is a rigid disc is invalid when converting an applied torque to a generated longitudinal force, even on a smooth road. The dynamics of the tyre need to be taken into account. This explains the poor performance seen by single point-follower contact methods employed by many semi-empirical tyre models when simulating tyres on rough roads. Zerophase low-pass filtering the measured tyre forces and brake moment improved the kinetic rolling radius calculation, but not sufficiently to justify the assumption that the wheel may be approximated as a rigid disc. 
A frequency domain analysis was performed and several observations were made. The vehicle's pitch natural frequency is at approximately $4 \mathrm{~Hz}$ and is excited during braking on any hard terrain. The wheel hop natural frequency may also be seen in the FFT magnitude of the vertical tyre force at $16 \mathrm{~Hz}$. A small peak in the 35 to $40 \mathrm{~Hz}$ range was seen on the longitudinal tyre force FFT magnitude plots; this corresponds to the tyre's torsional natural frequency.

It is of the utmost importance that designers working in the field of Advanced Driver Assist Systems (ADAS) take note of the problems identified by this study and refrain from using semi-empirical curve fit methods that rely on rigid body assumptions to model complex tyre dynamics, especially for rough road and off-road applications.

The scope of this paper was limited to investigating the rolling radius of a single tyre on hard terrain and this has barely scratched the surface of this hotly debated topic. Extending the approach used in this paper to the same tyre on deformable terrains such as sand and mud and on low friction terrains such as snow and ice may give more insight. Continuing the work on hard terrains at different tyre inflation pressures may also open up new areas of investigation.

The use of more complex models such as a rigid ring model or a brush tyre model has to be investigated. When moving towards more complex models, the definition of the rolling radius becomes problematic. These higher order models may be used in conjunction with state and parameter estimation techniques to directly estimate the longitudinal tyre slip and tyre friction. The measurement technique (DIC combined with a WFT) used in this study is a powerful tool that may be used to validate the performance of such estimators. If the development of a longitudinal tyre slip and tyre friction estimator is successful, the concept of a rolling radius may become unnecessary.

\section{REFERENCES}

ADCOX, J., AYALEW, B., RHYNE, T., CRON, S. \& KNAUFF, M. 2012. Interaction of anti-lock braking systems with tire torsional dynamics. Tire Science and Technology, Vol. 40, No. 3, pp.171-185.

BECKER, C. M. \& ELS, P. S. 2012. Wheel force transducer measurements on a vehicle in transit. 12th European Regional Conference of the ISTVS. Pretoria, South Africa.

BOSCH, H.-R. B., HAMERSMA, H. A. \& ELS, P. S. 2016. Parameterisation, validation and implementation of an all-terrain SUV FTire tyre model. Journal of Terramechanics, Vol. 67, pp.11-23.

BOSCH, R. 2005. Driving Stability Systems, Sae Soc Of Automotive Eng (SAE), Warrendale, Pennsylvania

BOTHA, T. R. \& ELS, P. S. Tire Longitudinal Slip-Ratio Measurement Using a Camera. ASME 2014 International Design Engineering Technical Conferences and Computers and Information in Engineering Conference, 2014. American Society of Mechanical Engineers, V003T01A043V003T01A043.

BOTHA, T. R., SHYROKAU, B., ELS, P. S. \& HOLWEG, E. 2015. Kinematic analysis of a tyre rolling over rough terrain using digital image correlation. In: PAOLUZZI, R. (ed.) 13th ISTVS European Conference ISTVS. Rome, Italy

BREUER, B. \& BILL, K. H. 2008. Brake technology handbook.

FISCHLER, M. A. \& BOLLES, R. C. 1981. Random sample consensus: a paradigm for model fitting with applications to image analysis and automated cartography. Communications of the ACM, 24, 381-395. 
GILLESPIE, T. D. 1992. Fundamentals of Vehicle Dynamics, Warrendale, PA, Society of Automotive Engineers, Inc.

HAMERSMA, H. A. \& ELS, P. S. 2014. Improving the braking performance of a vehicle with ABS and a semi-active suspension system on a rough road. Journal of Terramechanics, 56, 91-101.

KISS, P. 2003. Rolling Radii of a Pneumatic Tyre on Deformable Soil. Biosystems Engineering, 85, 153-161.

LOWE, D. G. 2004. Distinctive image features from scale-invariant keypoints. International journal of computer vision, 60, 91-110.

MERIAM, J. L. \& KRAIGE, L. G. 2012. Engineering mechanics: dynamics, John Wiley \& Sons.

MILLER, S. L., YOUNGBERG, B., MILLIE, A., SCHWEIZER, P. \& GERDES, J. C. Calculating longitudinal wheel slip and tire parameters using GPS velocity. American Control Conference, 2001. Proceedings of the 2001, 2001. IEEE, 1800-1805.

PENNY, W. C. W. \& ELS, P. S. 2016. The test and simulation of ABS on rough, non-deformable terrains. Journal of Terramechanics, 67, 1-10.

SIEGERT, E., VON GLASNER, E.-C., GEIßLER, H., VAN ZANTEN, A., BERG, P., BECKER, R., PFÄFFLE, J., SOWA, P., CZINCZEL, A., SCHMIDT, G., GERSTENMAIER, J., KNUST, A., KÜHNER, K., REINKE, K.-H., STEGMAIER, A., MEIßNER, M. \& SIGL, A. 1999. Drivingsafety Systems, Stuttgart, Germany, Robert Bosch GmbH.

UPADHYAYA, S., CHANCELLOR, W. \& WULFSOHN, D. 1988. Sources of variability in traction data. Journal of terramechanics, 25, 249-272. 